\author{
Proceedings of the $9^{\text {th }}$ International Conference on Applied Informatics \\ Eger, Hungary, January 29-February 1, 2014. Vol.1. pp.41-50 \\ doi: 10.14794/ICAI.9.2014.1.41
}

\title{
A new aspect of congestion detection over a large amount of real-time information*
}

\author{
Marcell Fekete, Zoltán Vincellér, Zoltán Ács
}

\author{
Eötvös Loránd University \\ feketem.77@gmail.com, vzoli@inf.elte.hu, acszolta@inf.elte.hu
}

\begin{abstract}
In these decades, we started to use such devices as mobile phones or GPS navigators, which are capable of recording geographical trajectories. However the available amount of information is usually far too large to analyze them with traditional algorithms, these kinds of data sources can hold a lot of useful information over the observed area. That is why we should use an efficient method or protocol to store and to process this kind of geographical information. The detection of congestions can help to optimize a traffic system by multiple ways. For example, this is the most straightforward way to detect over- and under loaded points in a system or to calibrate the traffic light to ease their load. By distinguishing specific patterns and tracking the movements of patterns, it is possible to discover the underlying reasons for congestion at certain points of traffic system. For instance, several minor congestions can lead to a major or multiple ones at different points in the system. The cause might be easier to eliminate than to break up the several or more complex congestions. By generalizing the definition of the congestion, the behavior of each object group takes shape. By analyzing these shapes and movement patterns -with the help of a specialist- a system could propose various investments' project to decrease the load on a heavily loaded traffic subsystem or simply to make the people live more comfortable. Such a system could be used by professionals at urban management and the system ultimately - with the support of a proper semantic database - can be fully automatized and integrated into the control system of a smart city solution. We adopted a simple clustering algorithm (DBScan[2]) and a method (AFOPT[3]), which mines various patterns from FCI data (GetMove) to detect such events. This paper presents a new model to compress and to process large amount of geographical information and architecture to detect typical traffic events (like congestions). It also proposes a method to understand trends in the behavior of objects by simplifying the data and using the
\end{abstract}

${ }^{*}$ The authors thank the partial support of the project GOP-1.1.1-11-2012-0416 supported by the European Union. 
aforementioned existing tools.

Keywords: data mining, pattern mining, congestion detection, human behavior

$M S C: 68 \mathrm{P} 05,68 \mathrm{Q} 6,68 \mathrm{~T} 10,68 \mathrm{~T} 30$

\section{Introduction}

The amount of spatiotemporal data regarding human movements is rapidly increasing within every minute. People share their whereabouts of their own free will. Moreover, in some countries they are willing to contribute their anonymized locations on any given time by signing their standard phone contract. From these spatiotemporal databases, we can characterize the traffic system of a city, and with these characteristics we are able to analyze certain aspects of a network to optimize them with data mining methods discussed in [4]. Although it is easy to see the benefits of these data, there are not many applications which utilize effectively them. Despite its usefulness, there are no widespread applications which could effectively utilize them. Before we start to optimize a system, we have to define several corresponding factors which make a system not-optimized. However it is not easy to find these factors.

A simple straightforward approach is to find such points, where congestions are often occurring. Let us analyze the crossroads a bit, where there are objects more than a certain threshold. This method holds some difficulties, such as: we cannot know whether an object is a car, or a pedestrian because of the anonymity. Big enough group of pedestrians can cause unwanted congestions. [6], [7] and [8] deals with the application of information regarding individual positions and with the problem of finding an effective integrated model. However its motivation is different, their results may be applied on this field as well. Another issue is that we cannot measure the spatial volume of a congestion. For example, let us take a crossroad which has two roads. One of them has one lane, and the other has two. Let the single lane be congested as much as it can be and let the double lane be completely free. In this case the crossroad has its capacity $1 / 3$ saturated. A method, with multiple thresholds, which could detect such an event, should give lots of false alarms as well. It may be also beneficial to determine the underlying connections between different congestions. By doing so, it may be possible to solve several minor congestions to ease a major one which would take much more effort to deal with. The objects behavior takes shape by detecting periodicity in the found congestion. Typically congestions may happen in certain times near shopping facilities. With proper tuning of the algorithm, even the starting and end points of the congested paths can be mined. With these in mind, an expert could lay out plans to construct or reconstruct facilities to distribute the load of the system. By doing so the systems overall stability is optimized and even part of the residents' everyday life may have become easier. In this paper we will propose a method to tackle the mentioned issues in congestion detection, mining different 
patterns in them and finally detecting periodicity in the mined patterns.

\section{Concept of periodic patterns}

On the level of abstraction, the traffic system of a city is represented by a graph. Each node represents a crossroad, while edges represent the roads. On this level there is no need for realism in the sense of curviness of the roads, what really matters is the fact that an event occurs on a certain vertex or edge.

\section{Graph representation of the network}

$\mathrm{G}\left(\mathrm{E}, \mathrm{V}, \mathrm{W}_{E}, \mathrm{~W}_{G}, \mathrm{~T}_{E}, \mathrm{~T}_{V}\right)$ is a network graph, where

- $\forall \mathrm{e} \in \mathrm{E}$ is an edge

- $\forall \mathrm{v} \in \mathrm{V}$ is a vertex

- $\forall \mathrm{w}_{e} \in \mathrm{W}_{E}$ is the weight (threshold) of edge e

- $\forall \mathrm{w}_{v} \in \mathrm{W}_{V}$ is the weight (threshold) of node $\mathrm{v}$

- $\forall \mathrm{T}_{a_{i}}$ is the set of objects on edge (vertex) a in time $\mathrm{i}$

- $\mathrm{T}_{e}=\cup\left\{\mathrm{T}_{e_{i}}\right\}$

- $\mathrm{T}_{E}=\cup\left\{\mathrm{T}_{e}\right\}$

- $\mathrm{T}_{v}=\cup\left\{\mathrm{T}_{v_{i}}\right\}$

- $\mathrm{T}_{V}=\cup\left\{\mathrm{T}_{v}\right\}$

According to the definition, the network itself contains the informations regarding the general whereabouts of every object in the system for every timestep. In most cases the information about their number is sufficient:

Definition 2.1 (Load). $\mathrm{L}(\mathrm{a}, \mathrm{i}):=\left|\mathrm{T}_{a i}\right|$, is the load of element a in time $\mathrm{i}$.

Let a node or edge be congested if its' load is more than the corresponding weight. At first it seems as if it inherited all of the difficulties mentioned in the introduction, but it is not. The congestion property is defined on both edges and vertexes, so two non-congested edges' intersection can be a congested node, and respectively a congested and non-congested edge can have a non-congested node. This model fits the expectations we defined. The main weakness, as it is, the detected congestions are detached.

Any congestion can be represented by the objects which cause them and with the time of occurrence. None of the objects can be at two different places in the same time, so this statements stands.

According to this statement, any set of objects has a set of timestamps and for every timestamp these objects participates in the same congested element of the network. 
Definition 2.2 (Congestion). (O,T) is a congestion if

- $\forall \mathrm{o} \in \mathrm{O}$ is an object

- $\forall \mathrm{t} \in \mathrm{T}$ is a timestamp

- $\forall \mathrm{t} \in \mathrm{T}, \forall \mathrm{o} \in \mathrm{O}, \exists \mathrm{a} \in \mathrm{E} \cup \mathrm{V}$ : $\mathrm{o} \in \mathrm{T}_{a_{t}} \wedge \mathrm{L}(\mathrm{a}, \mathrm{i})>\mathrm{w}_{a}$

- $|\mathrm{T}|>0$

- $\mathrm{T}$ is the occurrence set

- $\mathrm{O}$ is the object set

Definition 2.3 (Frequency). $\operatorname{Frq}(\mathrm{O}, \mathrm{T}):=|\mathrm{T}|$

Definition 2.4 (Support) $\sigma(\mathrm{O}, \mathrm{T}):=|\mathrm{O}|$

A subset of the occurrences of a given congestion, with the same object set forms a pattern. Congestions can be classified by their frequency, support and by its' occurrence set traits. Spatiotemporal patterns are designed to define group of objects, with similar movement behavior in a time interval. Many different kind of pattern were proposed. In this paper we discuss swarms, closed swarms, group patterns and periodic patterns [1].

Definition 2.5 (Swarm). A pair $(\mathrm{O}, \mathrm{T})$ is a swarm if:

- $(\mathrm{O}, \mathrm{T})$ is a pattern

- $\operatorname{Frq}(\mathrm{O}, \mathrm{T})>\min _{t}$

- $\sigma(\mathrm{O}, \mathrm{T})>\varepsilon$

A swarm follows a group of objects in time. This pattern can show how does the same group of objects affects the overall system stability. Formally it is sufficient to be a pattern, but in real application there is no point to doing so. To match the needs of the application with the theory there is a closed definition of the swarm pattern.

Definition 2.6 (closed swarm). A pair $(\mathrm{O}, \mathrm{T})$ is a closed swarm if:

- $(\mathrm{O}, \mathrm{T})$ is a swarm

- $\nexists \mathrm{O}^{\prime}: \mathrm{O} \subset \mathrm{O}^{\prime}$ and $\left(\mathrm{O}^{\prime}, \mathrm{T}\right)$ is swarm

- $\nexists \mathrm{T}^{\prime}: \mathrm{T} \subset \mathrm{T}^{\prime}$ and $\left(\mathrm{O}, \mathrm{T}^{\prime}\right)$ is swarm

The closed swarm pattern is maximal in the size of both $\mathrm{O}$ and $\mathrm{T}$ sets, so potentially it holds the most possible information. 
Theorem 2.7. Every closed swarm is a congestion, but not every congestion is a closed swarm.

Proof. A closed swarm is a pattern. Due to the closure in time, this pattern has to be maximal, which is the original congestion.

In the definition of congestion, there is no maximal requirement, so for every $(\mathrm{O}, \mathrm{T})$ closed swarm there is $|\mathrm{P}(\mathrm{T})|-1$ other congestion, where $\mathrm{P}(\mathrm{T})$ is the power set of $\mathrm{T}$ set.

On extended time intervals, even the closed swarms, becomes redundant, due to periodicity. In this abstract model, the redundancy can be is easily extracted from the time series. In section 4 we will discuss in detail the projection between the applied and the abstract model.

\section{Methods for detecting congestions}

In the applied informatics, it is inefficient and importable to store the whole network graph, if it is not necessary. In this section we will propose a method to detect congestions and patterns, without storing any data about the network itself. The only available information is about the objects whereabouts of each object in every time-step. Usually there are much more time-step then object. For efficiency we process the data on each layer in time, then we apply the costly analysis between these layers.

As the abstract model shows, the patterns can be classified by both their frequency and their support. During the process there is a filter for congestions with low frequency. Potentially, they do not hold many information and treated as noise, which holds back the costly process' efficiency.

\subsection{Congestion detection}

Since there is no data about the network itself, the definition of congestion must be derived from the objects interdependent behaviors. In every time-step only the geographical locations of the objects is known, hence it seems inevitable to analyze them by their distance from each other. We adopted the DBScan (Density Based Scan[2]) algorithm, which has the following steps:

Given the set $\mathrm{O}_{d b}$, which have the objects with their respective locations and the $\mathrm{C}_{d b}$, which contains the found congestions. It also has two parameters, eps and $\mathrm{N}$. It selects and removes a point from the database, and collects and removes every point in its eps distance vicinity. If there are more than $\mathrm{N}$ collected point, then it puts them into a set $\mathrm{C}$. for every point of $\mathrm{C}$ it recursively collects and removes every point which is closer then eps to at least one of C's points. I C can't be expanded, it puts $\mathrm{C}$ into $\mathrm{C}_{d b}$. This procedure is iterated until $\mathrm{O}_{d b}$ is empty.

The resulting congestions are represented by its' members and by the time of occurrence, just as it is in the abstract model, hence this method is compatible with the model. If the graph network has a function which generates the weights 
of each element according to its' capacity, there can be found a G graph with the weights which represents these results. In this paper we assume the presence of such function.

In the future we are planning to experiment the effect of a more complex clustering method, discussed in [5].

\subsection{Pattern mining}

At this point there is no need to store each individual time for the congestion.

Definition 3.1 (Itemset). $\hat{\mathrm{I}}:=\{1 . . n\}$ set of objects which participates in a congestion. Each object has an integer as its' unique ID.

Even without the times stored, there are multiple instances of the same congestion.

Definition 3.2 (transactions set).

$\mathrm{T}:=\left\{\mathrm{t}_{1} . . \mathrm{t}_{m}\right\}: \mathrm{t}_{i} \in \hat{\mathrm{I}}$

$\mathrm{T}(\mathrm{P}):=\left\{\mathrm{t}_{1} . . \mathrm{t}_{n}\right\}: \mathrm{t}_{j} \in \mathrm{P}$

An itemset and its' transactions set only lacks the uniqueness of each occurrences. Since none of the abstract model makes use of that, the pair $(\hat{\mathrm{I}}, \mathrm{T})$ will do as congestions with their every merit exploited. This representation is more compact, because there is limitation to the elements of the transactions set aside from the fact, that every element has $\mathrm{P}$ as its' subset.

The two used trait is as follows:

Definition 3.3 (frequency). $\operatorname{Frq}(\mathrm{P}):=|\mathrm{T}(\mathrm{P})|$

Definition 3.4 (support). $\sigma(\mathrm{P}):=|\mathrm{P}|$

An itemset called frequent itemset, if its' frequency is greater than a predefined threshold. It also has a closure property:

Definition 3.5 (closed itemset). $\mathrm{P}$ is closed if and only if,

$$
\left[\mathrm{P} \subseteq \mathrm{T}(\mathrm{P}) \rightarrow \neg\left(\exists \mathrm{P}^{\prime}\right): \mathrm{T}(\mathrm{P})=\mathrm{T}\left(\mathrm{P}^{\prime}\right) \wedge \mathrm{P} \subset \mathrm{P}^{\prime}\right]
$$

With this applied terminology, [Get_Move] defined the swarm and closed swarm patterns as follows:

Definition 3.6 (swarm). (P, T) is swarm, if

- $\mathrm{T} \subseteq \mathrm{T}(\mathrm{P})$

- $\sigma(\mathrm{P})>\varepsilon$

- $\operatorname{Frq}(\mathrm{P})>\min _{t}$

Definition 3.7 (closed swarm). A pair $(\mathrm{P}, \mathrm{T})$ is a closed swarm if: 
- $(\mathrm{P}, \mathrm{T})$ is a swarm

- $\nexists \mathrm{P}^{\prime}: \mathrm{P} \subset \mathrm{P}^{\prime}$ and $\left(\mathrm{P}^{\prime}, \mathrm{T}\right)$ is swarm

- $\nexists \mathrm{T}^{\prime}: \mathrm{T} \subset \mathrm{T}^{\prime}$ and $\left(\mathrm{P}, \mathrm{T}^{\prime}\right)$ is swarm

By the definition of the swarm pattern, every frequent itemset, which satisfies the support condition is swarm. As the next theorem shows, they also inherit the connection between closed swarms and closed itemsets.

Theorem 3.8. Every frequent closed itemset (FCI) is a closed swarm on its own.

Proof. The frequency condition utilizes the same $\varepsilon$, therefore we will show, that the two closure property has the same meaning.

Let $\mathrm{P}$ be a closed itemset. By definition, there is no superset of $\mathrm{P}$, which has the same set of occurrences, so $\mathrm{P}=\cap \mathrm{T}(\mathrm{P})$. This essentially means that, $\mathrm{P} \cup\{\mathrm{a}\}=\cap \mathrm{T}$, where $\mathrm{T} \subset \mathrm{T}(\mathrm{P})$, which violates the condition of closed swarm.

With the applied model, the task of pattern mining is projected to the frequent closed itemset mining problem.

\section{Detecting periodicity}

Although in the abstract model it's simple to detect periodic patterns, unfortunately in the applied model it is not the case. First of all, we have no information about the network, it is impossible to determine whether the measured positions are on the same lane or not. On the other hand, it would be costly to check every occurrence of every member of the congestion and analyze their interdependent positions. Therefore we propose a classification method which precedes the congestion detection, and introduces periodic points in the system.

\subsection{Pre-classification}

The proposed pre-classification algorithm sweeps through each objects every position in space and time. The basic idea is to clean all of the objects from the database, which is sure to be non-periodic and keep the ones with certain periodic properties. Let a position of a given object be a periodic occurrence candidate, if it is close enough to another position in another time of the same object, which is a far enough time from the examined spatiotemporal point. If the predefined distance in space is not large enough, there may be cases, when different periods interests or even have complete containing relationship. To tackle this problem, a simple FCI mining sweep can be applied. Although the cost of this check is sufficiently low, we implemented a greedy algorithm as follows:

$\underline{\text { Periodic }}(\mathrm{X}$ : set of spatiotemporal points of the same object, Y: output, time_TH: minimum time between candidates, space_TH: maximal distance in space between candidates, length_TH: minimum period length) 
1. foreach $\mathrm{x}$ from $\mathrm{X}$

2. let $\mathrm{Z}$ be an empty set

3. remove $\mathrm{x}$ from $\mathrm{X}$

4. put $\mathrm{x}$ to $\mathrm{Z}$

5. make the set of points candidates which is closer in space than space_ $\mathrm{TH}$

6. let $\mathrm{t}$ be the time of occurrence of $\mathrm{x}$

7. while $\mid$ candidates $\mid>0$

8. Greedy-shrink(time_TH, t, x, candidates, X, Z)

9. if $|\mathrm{Z}|>$ length_TH

10. put $\mathrm{Z}$ to $\mathrm{Y}$

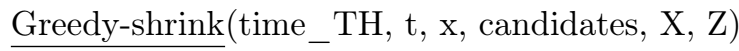

1. remove every point from candidates which is closer in time than time_TH

2. let a be the closest point in space to $\mathrm{x}$

3. remove a from a from both candidates and $\mathrm{X}$

4. let $\mathrm{t}$ be the time of occurrence of a

5. put a to $\mathrm{Z}$

The method is greedy, because if an element were a candidate once, it won't examine it again. With this, there can't be any intersection between the periods. The other version of the algorithm, won't delete anything from the set X. With the non-greedy algorithm, the method which leads to a wider set of results, with possibly other kind of periodic properties.

\section{Results}

Experiments were conducted on both artificial and real life data. As for artificial data, we used an open-source simulator software, called SUMO. Experiments on this dataset show that, the algorithm - as a whole - is fairly efficient, even on non-divided architectures.

The simulation used the topology of Budapest, with 845 objects moving in 1200 time-steps.

The pre-classification were able to detect the periodic patterns defined in this paper on the simulated data and were able to reduce the cost of the process. After the results on the simulated data, we deemed to be ready for real life tests. We 


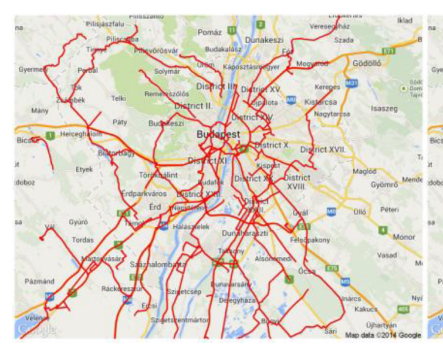

(a)

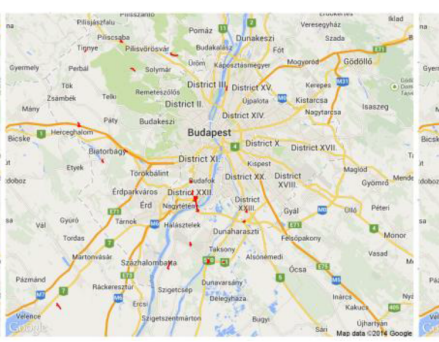

(b)

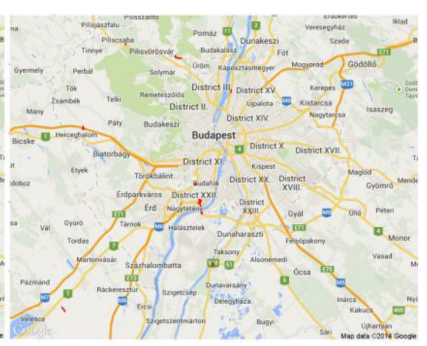

(c)

Figure 1: (a) shows all of the objects with all of their occurrences. After the classification there are significantly fewer occurrences as it can be seen on (b). (c) is the result of the proposed algorithm

used real life FCD data to do so. We also limited these data to Budapest, to be able to compare to the experiments on artificial data.

As the figure shows, the pre-classification can immensely reduce the amount of data, by discarding the non-periodic records.

The presented method was able to detect periodic congestions, which holds significant and lasting impact on the flow of the whole system.

\section{Conclusion}

The execution time of the implemented algorithm strongly depends on the amount of the input data. Our proposed classification filters out the periodic points from the input data. In practice, there are usually much more non-periodic points than periodic. That is the reason why, our classification is able to greatly reduce the size of the input data, therefore the overall execution time is decreased as well.

Traditional methods scan through the resulting patterns to detect periodicity. They tend to scale with the amount and length of the resulting patterns quadratic. In comparison, our algorithm scales with amount the objects, and their average length of their occurrences. Therefore the cost of our method is usually lower than the cost of the traditional methods.

Recently the amount of works regarding smart cities is ever increasing. The proposed algorithm could support the decision making system of a smart cities. We will continue our work to make tools and applications to help both the current expert-led systems and the progress smart cities.

\section{References}

[1] Phan Nhat Hai, Pascal Poncelet, Maguelonne Teisseire, „GeT_Move: An Efficient and Unifying Spatio-Temporal Pattern Mining Algorithm for Moving Objects", IDA'12 
Proceedings of the 11th international conference on Advances in Intelligent Data Analysis

[2] Ester M., Kriegel H.-P., Sander J., Xu X. A, „Density-Based Algorithm for Discovering Clusters in Large Spatial Databases with Noise", KDD '96, Portland, pp. 226-231.

[3] G. Liu, H. Lu, J. X. Yu, W. Wang, X. Xiao. AFOPT: An Efficient Implementation of Pattern Growth Approach. The 1st International Workshop on Frequent Itemset Mining Implementations (FIMI'03), Melbourne, Florida, USA, 2003.

[4] Adriaans, Pieter, and Dolf Zantinge. "Adatbányászat." (Translator: Attila Kiss) Panem Könyvkiadó, Budapest (2002).

[5] Vathy-Fogarassy, Agnes, Attila Kiss, and Janos Abonyi. "Hybrid minimal spanning tree and mixture of Gaussians based clustering algorithm." Foundations of Information and Knowledge Systems. Springer Berlin Heidelberg, 2006. 313-330.

[6] Matuszka, Tamás, Gergő Gombos, and Attila Kiss. "A New Approach for Indoor Navigation Using Semantic Webtechnologies and Augmented Reality." Virtual Augmented and Mixed Reality. Designing and Developing Augmented and Virtual Environments. Springer Berlin Heidelberg, 2013. 202-210.

[7] Tamás Matuszka, Attila Kiss: Geodint: Towards Semantic Web-Based Geographic Data Integration. ACIIDS (1) 2014: 191-200

[8] Csaba István Sidló: Entity Resolution with Heavy Indexing. ADBIS (2) 2011: 137-146 